\title{
The quantum discrete self-trapping equation in the Hartree approximation
}

\author{
Ewan Wright ${ }^{a}$, J.C. Eilbeck ${ }^{b}$, M.H. Hays ${ }^{c}$, P.D. Miller ${ }^{c}$ and A.C. Scott ${ }^{c}$ \\ aptical Sciences Center and Department of Physics, University of Arizona, Tucson, AZ 85721, USA \\ b Department of Mathematics, Heriot-Watt University, Riccarton, Edinburgh, EH14 4AS, UK \\ c Department of Mathematics, University of Arizona, Tucson, AZ 85721, USA
}

Received 19 February 1993

Revised manuscript received 21 June 1993

Accepted 24 June 1993

Communicated by $\mathbf{H}$. Flaschka

\begin{abstract}
We show how the Hartree approximation (HA) can be used to study the quantum discrete self-trapping (QDST) equation, which - in turn - provides a model for the quantum description of several interesting nonlinear effects such as energy localization, soliton interactions, and chaos. The accuracy of the Hartree approximation is evaluated by comparing results with exact quantum mechanical calculations using the number state method. Since the Hartree method involves solving a classical DST equation, two classes of solutions are of particular interest: (i) Stationary solutions, which approximate certain energy eigenstates, and (ii) Time dependent solutions, which approximate the dynamics of wave packets of energy eigenstates. Both classes of solution are considered for systems with two and three degrees of freedom (the dimer and the trimer), and some comments are made on systems with an arbitrary number of freedoms.
\end{abstract}

\section{Introduction}

Consider the classical discrete self-trapping (DST) equation, which can be written in the form [1,2]

$$
\mathrm{i} \frac{\mathrm{d} A_{j}}{\mathrm{~d} t}-\omega_{0} A_{j}+\sum_{k=1}^{f} m_{j k} A_{k}+\gamma\left|A_{j}\right|^{2} A_{j}=0,
$$

where $j=1,2, \ldots, f$ counts the number of freedoms, $\omega_{0}$ is the site frequency, and the $A_{j}$ 's are complex mode amplitudes. Also $M=\left[m_{i j}\right]$ is an $f \times f$ symmetric matrix with real coefficients $m_{j k}=m_{k j}$ and $m_{j j}=0$ describing linear coupling between identical oscillators at the $j^{\text {th }}$ and $k^{\text {th }}$ freedoms, and $\gamma$ is a nonlinear or anharmonic parameter for each individual oscillator. This system has applications to molecular crystals, molecular dynamics, nonlinear optics, and biomolecular dynamics; see [1] for a list of references.

As a model of identical molecular stretching oscillators, $A_{j}=\left(x_{j} \sqrt{k}+\mathrm{i} \dot{x}_{j} \sqrt{m}\right) / \sqrt{2 \omega_{0}}$ is the complex mode amplitude of the $j$ th oscillator (where $\omega_{0}=\sqrt{k / m}$ and $k$ and $m$ are the linear spring constant and reduced mass of an oscillator) [3].

Under quantization $A_{j}\left(A_{j}^{*}\right) \rightarrow b_{j}\left(b_{j}^{\dagger}\right)$, the standard boson lowering (raising) operators, and the classical Hamiltonian becomes the energy operator [4,5] 


$$
\widehat{H}=\sum_{j=1}^{f}\left[\left(\omega_{0}-\gamma\right) b_{j}^{\dagger} b_{j}-\frac{1}{2} \gamma b_{j}^{\dagger} b_{j}^{\dagger} b_{j} b_{j}\right]-\sum_{j \neq k} m_{j k} b_{j}^{\dagger} b_{k},
$$

where zero point energy has been neglected.

Although exact eigenfunctions of $\widehat{H}$ can be constructed for any finite number $(n)$ of bosons [4,5], this becomes technically inconvenient when $n$ is large and $f \geqslant 3$. In such cases it is interesting to consider approximate methods. The method discussed here is the Hartree approximation [6], which - in essence - determines the behavior of each boson in the presence of the $n-1$ others. An exact analysis, called the number state method (NSM) [4,5] is used to determine the accuracy of various Hartree results.

It is interesting and important to observe that the equation to be solved in order to construct the Hartree approximate wave function is almost identical to the motivating classical equation (1.1). Thus the classical dynamics is closely related to the quantum dynamics, at least in the Hartree approximation.

Some general aspects of the quantum analysis of $\widehat{H}$ are presented in the following section, and the Hartree approximation is described in detail in section 3 . Here we stress the close connection between the Hartree approximate eigenfunctions of $\hat{H}$ in eq. (1.2) and solutions of the classical DST in eq. (1.1). In section 4 we consider quantum expectation values and energies of stationary states. As examples, we discuss the dimer system $(f=2)$ in section 5 and the trimer system $(f=3)$ in section 6. In both examples we consider the significance of Hartree wave functions that are based upon stationary solutions of the classical DST and those that are based upon time dependent solutions. Some comments on the accuracy of the Hartree approximation in systems with an arbitrary number of freedoms are presented in section 7 , and conclusions are summarized in section 8 . Throughout the paper we assume $\hbar$ to be unity.

\section{Quantum analysis}

Our analysis of $\widehat{H}$ is in the Schrödinger picture; thus the state vector $|\psi(t)\rangle$ is time dependent, and the quantum operators are those at time $t=0$. The Schrödinger equation for the state vector is then

$$
\mathrm{i} \frac{\mathrm{d}}{\mathrm{d} t}|\psi(t)\rangle=\widehat{H}|\psi(t)\rangle
$$

A general $n$-boson state vector can be expanded in the Fock space as [7]

$$
\left|\psi_{n}(t)\right\rangle=\frac{1}{\sqrt{n !}} \sum_{j_{1}=1}^{f} \sum_{j_{2}=1}^{f} \ldots \sum_{j_{n}=1}^{f} \theta_{n}\left(j_{1}, j_{2}, \ldots j_{n}, t\right) b_{j_{1}}^{\dagger} b_{j_{2}}^{\dagger} \ldots b_{j_{n}}^{\dagger}|0\rangle
$$

where $|0\rangle \equiv|0\rangle_{1}|0\rangle_{2} \cdots|0\rangle_{f}$ is the vacuum state. The $\theta_{n}$ are $f^{n}$ time dependent coefficients of corresponding number states. For example if $f=2$ and $n=3, \theta_{3}(2,1,2, t)$ indicates that the first boson is put onto the second freedom, the second boson is put on the first freedom, and the third boson is put on the second freedom; thus it is a coefficient of the number state $|1\rangle|2\rangle$. More generally, $\theta_{n}\left(j_{1}, j_{2}, \ldots j_{n}, t\right)$ is the $n$-boson wave function, which is normalized as 


$$
\sum_{j_{1}=1}^{f} \sum_{j_{2}=1}^{f} \cdots \sum_{j_{n}=1}^{f}\left|\theta_{n}\left(j_{1}, j_{2}, \ldots j_{n}, t\right)\right|^{2}=1 .
$$

Substituting the state vector in eq. (2.2) into the Schrödinger equation in eq. (2.1) and using the boson commutation relations $\left[b_{j}, b_{k}^{\dagger}\right]=\delta_{j k}$, we obtain the following Schrödinger equation for the $n$-boson wave function:

$$
\begin{aligned}
& \left(\mathrm{i} \frac{\mathrm{d}}{\mathrm{d} t}-n \widetilde{\omega}_{0}\right) \theta_{n}\left(j_{1}, j_{2}, \ldots, j_{n}, t\right)+\sum_{k=1}^{f}\left[m_{j_{1}, k} \theta_{n}\left(k, j_{2}, j_{3}, \ldots, j_{n}, t\right)\right. \\
& \left.\quad+m_{j_{2}, k} \theta_{n}\left(j_{1}, k, j_{3}, \ldots, j_{n}, t\right)+\cdots+m_{j_{n}, k} \theta_{n}\left(j_{1}, j_{2}, \ldots, k, t\right)\right] \\
& \quad+\gamma \sum_{l=1}^{n} \sum_{m>l}^{n} \delta_{j_{l}, j_{m}} \theta_{n}\left(j_{1}, \ldots, j_{l}, \ldots, j_{m}, \ldots, j_{n}, t\right)=0,
\end{aligned}
$$

where $\widetilde{\omega}_{0} \equiv\left(\omega_{0}-\gamma\right)$. Eq. (2.4) is the Schrödinger equation for a system of bosons at $f$ discrete sites (freedoms) with linear coupling $\left(m_{j k}\right)$ and a Kronecker delta-function interaction between pairs of bosons. It can be compared with the corresponding quantum field theory for a Bose gas, which involves a Dirac delta function interaction [7].

As was noted above, the $\theta_{n}$ 's in eq. (2.2) are $f^{n}$ time dependent coefficients of corresponding number states, but not all are independent since bosons are indistinguishable. For example if $f=2$ and $n=2$, eq. (2.2) becomes

$$
\left.\left|\psi_{2}(t)\right\rangle=\theta_{2}(1,1, t)|2\rangle|0\rangle+\sqrt{1 / 2}\left[\theta_{2}(1,2, t)+\theta_{2}(2,1, t)\right)\right]|1\rangle|1\rangle+\theta_{2}(2,2, t)|0\rangle|2\rangle,
$$

while in the number state method $[4,5]$ the most general eigenfunction of the boson number operator is written as

$$
\left|\psi_{2}\right\rangle=c_{1}|2\rangle|0\rangle+c_{2}|1\rangle|1\rangle+c_{3}|0\rangle|2\rangle,
$$

and $c_{1}, c_{2}$ and $c_{3}$ are then chosen so $\left|\psi_{2}\right\rangle$ is also an eigenstate of $\hat{H}$ with eigenvalue $E$. Time dependence is then introduced by multiplying each energy eigenfunction by the factor $\exp (-\mathrm{i} E t)$.

In eq. $(2.5), \theta_{2}(1,2, t)$ is equal to $\theta_{2}(2,1, t)$ because there is no physical difference between putting the first boson on the first freedom and the second on the second and putting the first on the second and the second on the first. Thus the order of the system is no larger than the number of ways, $p$, that $n$ bosons can be put on $f$ freedoms or

$$
p=\frac{(f+n-1) !}{n !(f-1) !}<f^{n} .
$$

If $\widehat{H}$ has additional symmetries, the order of the system to be solved may be less than $p$.

\section{Hartree approximation (HA)}

Since the order, $p$ in eq. (2.7), may be inconveniently large, we turn to the Hartree approximation (HA). This approximation is well known in quantum field theory [6] - in particular, nuclear many 
body theory and more recently to nonlinear optics [8] - but to our knowledge it has not yet been applied to a discrete system such as the QDST equation.

In the HA it is assumed that the $n$-boson wave function $\theta_{n}\left(j_{1}, \ldots, j_{n}, t\right)$ can be written as a product of the form

$$
\theta_{n}^{(\mathrm{H})}\left(j_{1}, \ldots, j_{n}, t\right)=\prod_{k=1}^{n} \Phi_{n, j_{k}}(t),
$$

which satisfies the symmetry condition for a many-boson boson wave function

$$
\theta_{n}\left(j_{1}, \ldots, j_{l}, \ldots, j_{m}, \ldots, j_{n}, t\right)=\theta_{n}\left(j_{1}, \ldots, j_{m}, \ldots, j_{l}, \ldots, j_{n}, t\right) .
$$

The basic idea is that each boson feels the same mean field potential due to all the other bosons, and the many-body wave function can be approximated as a product of single-boson wave functions $\Phi_{n, j_{k}}(t)$ with $j_{k}=1,2, \ldots, f$ and $k=1,2, \ldots, n$ labeling the boson. Since these single-boson wave functions are independent of $k$, we write them simply as $\Phi_{n, j}(t)$, where $j=1,2, \ldots, f$.

Using the HA wave function in eq. (3.1) the $n$-boson state vector in eq. (2.2) becomes

$$
\left|\psi_{n}(t)\right\rangle^{(\mathrm{H})}=\frac{1}{\sqrt{n !}}\left(\sum_{j=1}^{f} \Phi_{n, j}(t) b_{j}^{\dagger}\right)^{n}|0\rangle,
$$

and from eq. (2.3) the normalization condition is

$$
\sum_{j=1}^{f}\left|\Phi_{n, j}(t)\right|^{2}=1
$$

To obtain an equation of motion for $\Phi_{n, j}(t)$ we note that eq. (2.4) for $\theta_{n}\left(j_{1}, j_{2}, \ldots, j_{n}, t\right)$ can be obtained by extremizing the functional

$$
\begin{aligned}
S= & \int_{-\infty}^{\infty} \mathrm{d} t \sum_{j_{1}=1}^{f} \sum_{j_{2}=1}^{f} \ldots \sum_{j_{n}=1}^{f} \theta_{n}^{*}\left[\left(\mathrm{i} \frac{\mathrm{d}}{\mathrm{d} t}-n \widetilde{\omega}_{0}\right) \theta_{n}\left(j_{1}, j_{2}, \ldots, j_{n}, t\right)\right. \\
& +\sum_{k=1}^{f}\left[m_{j_{1}, k} \theta_{n}\left(k, j_{2}, j_{3}, \ldots, j_{n}, t\right)+m_{j_{2}, k} \theta_{n}\left(j_{1}, k, j_{3}, \ldots, j_{n}, t\right)+\ldots\right. \\
& \left.\left.+m_{j_{n}, k} \theta_{n}\left(j_{1}, j_{2}, \ldots, k, t\right)\right]+\gamma \sum_{l=1}^{n} \sum_{m>l}^{n} \delta_{j_{l}, j_{m}} \theta_{n}\left(j_{1}, \ldots, j_{l}, \ldots, j_{m}, \ldots, j_{n}, t\right)\right]
\end{aligned}
$$

as $\delta S / \delta \theta^{*}=0$. By substituting the HA wave function from eq. (3.1) into eq. (3.4) and using the normalization condition of eq. (2.3) we obtain

$$
S^{(\mathrm{H})}=n \int_{-\infty}^{\infty} \mathrm{d} t \sum_{j=1}^{f}\left[\Phi_{n, j}^{*}\left(\mathrm{i} \frac{\mathrm{d} \Phi_{n, j}}{\mathrm{~d} t}-\widetilde{\omega}_{0} \Phi_{n, j}+\sum_{k=1}^{f} m_{j k} \Phi_{n, k}\right)+\frac{1}{2} \gamma(n-1)\left|\Phi_{n, j}\right|^{4}\right],
$$

where we exclude the degenerate case in which all the $m_{j k}$ are zero for some particular value of $j$. Requiring $\delta S^{(\mathrm{H})} / \delta \Phi_{n, j}^{*}=0$ for the optimal Hartree solution we obtain the following equation for the effective single-boson wave function: 


$$
\mathrm{i} \frac{\mathrm{d} \Phi_{n, j}}{\mathrm{~d} t}-\widetilde{\omega}_{0} \Phi_{n, j}+\sum_{k=1}^{f} m_{j k} \Phi_{n, k}+\gamma(n-1)\left|\Phi_{n, j}\right|^{2} \Phi_{n, j}=0 .
$$

Equation (3.6) is the main result of this section. Together with eq. (3.1) it enables us to construct $\theta_{n}^{(\mathrm{H})}\left(j_{1}, \ldots, j_{n}, t\right)$, the HA to the many-boson wave function $\theta_{n}\left(j_{1}, \ldots, j_{n}, t\right)$. In effecting this construction it is interesting to notice that Equation (3.6) is closely related to the classical DST in eq. (1.1). The differences are these:

i) The solution of eq. (3.6) is constrained by the normalization condition of eq. (3.3),

ii) The nonlinear parameter $\gamma$ is multiplied by the factor $(n-1)$, and

iii) The site frequency in eq. (1.1) has changed from $\omega_{0}$ to $\widetilde{\omega}_{0} \equiv \omega_{0}-\gamma$ in eq. (3.6).

Finally we note that eq. (3.6) can be written in Hamiltonian form as id $\Phi_{n, j} / \mathrm{d} t=\partial h_{n} / \partial \Phi_{n, j}^{*}$, where

$$
h_{n}=\sum_{j=1}^{f}\left(\tilde{\omega}_{0}\left|\Phi_{n, j}\right|^{2}-\frac{1}{2} \gamma(n-1)\left|\Phi_{n, j}\right|^{4}-\sum_{k=1}^{f} \Phi_{n, j}^{*} m_{j k} \Phi_{n, k}\right)
$$

is the effective single-boson Hamiltonian for one boson in the presence of the other $(n-1)$ bosons.

\section{Quantum expectation values}

The approximate Hartree state vector $\left|\psi_{n}(t)\right\rangle^{(\mathrm{H})}$ can now be used to calculate quantum expectation values. For example, the mean number of bosons on the $j^{\text {th }}$ freedom is

$$
\left\langle n_{j}(t)\right\rangle^{(\mathrm{H})}={ }^{(\mathrm{H})}\left\langle\psi_{n}(t)\left|b_{j}^{\dagger} b_{j}\right| \psi_{n}(t)\right\rangle^{(\mathrm{H})},
$$

and using eq. (3.2) in eq. (4.1) we obtain

$$
\left\langle n_{j}(t)\right\rangle^{(\mathrm{H})}=n\left|\Phi_{n, j}(t)\right|^{2} .
$$

Thus quantum expectation values can be related to solutions of eq. (3.6), which is identical in form to the classical DST in eq. (1.1). This is the basic reason for the usefulness of the HA: it connects expectation values of the quantum problem with solutions of the corresponding DST equation.

The Hartree approximation can also be used to obtain energies of stationary solutions of the QDST equation. Consider a stationary solution of eq. (3.6) with the form

$$
\Phi_{n, j}=\mathrm{e}^{-\mathrm{i} \Omega t} \chi_{n, j}
$$

The single-boson Hamiltonian defined in eq. (3.7) gives the energy of a single boson as

$$
e_{n}=\sum_{j=1}^{f}\left(\tilde{\omega}_{0}\left|\chi_{n, j}\right|^{2}-\frac{1}{2} \gamma(n-1)\left|\chi_{n, j}\right|^{4}-\sum_{k=1}^{f} \chi_{n, j}^{*} m_{j k} \chi_{n, j}\right) .
$$

For such stationary solutions the HA wave function in eq. (3.2) becomes

$$
\left|\psi_{n}(t)\right\rangle^{(\mathrm{H})}=\mathrm{e}^{-\mathrm{i} n \Omega t} \frac{1}{\sqrt{n !}}\left(\sum_{j=1}^{f} \chi_{n, j}(t) b_{j}^{\dagger}\right)^{n}|0\rangle,
$$

where $\Omega$ and $\chi_{n, j}$ are solutions of the nonlinear eigenvalue equation 


$$
\left(\Omega-\tilde{\omega}_{0}\right) \chi_{n, j}+\sum_{k=1}^{f} m_{j k} \chi_{n, k}+\gamma(n-1)\left|\chi_{n, j}\right|^{2} \chi_{n, j}=0 .
$$

The corresponding Hartiee energy for the $n$-boson system is then given by

$$
E_{n}^{(\mathrm{H})}={ }^{(\mathrm{H})}\left\langle\psi_{n}(\dot{t})|\widehat{H}| \psi_{n}(t)\right\rangle^{(\mathrm{H})},
$$

where $\widehat{H}$ is defined in eq. (1.2). Substituting eq. (4.5) into eq. (4.6), we find that

$$
E_{n}^{(\mathrm{H})}=n e_{n} .
$$

Thus in the HA the energy of an $n$-boson stationary state is just $n$ times the energy of a single-boson stationary state.

\section{The DST dimer $(f=2)$}

In this section we assume the renormalized site frequency $\widetilde{\omega}_{0} \equiv \omega-\gamma$ to be zero. With two freedoms $(f=2)$ and $m_{12} \equiv \epsilon$, the Hamiltonian operator in eq. (1.2) becomes

$$
\widehat{H}=-\sum_{j=1}^{2}\left[\epsilon b_{j}^{\dagger}\left(b_{j+1}+b_{j-1}\right)+\frac{1}{2} \gamma b_{j}^{\dagger} b_{j}^{\dagger} b_{j} b_{j}\right] .
$$

Our aim is to explore the conditions under which the Hartree wave function is or is not a useful approximation to the exact wave function. Using the number state method [4,5], energy eigenvalues are eigenvalues of the $(n+1) \times(n+1)$ tridiagonal matrix

$$
H_{n}=\left(\begin{array}{cccccc}
p_{1} & q_{1} & & & & \\
q_{1} & p_{2} & q_{2} & & & \\
& q_{2} & p_{3} & q_{3} & & \\
& & \ddots & \ddots & \ddots & \\
& & & q_{2} & p_{2} & q_{1} \\
& & & & q_{1} & p_{1}
\end{array}\right)
$$

where

$$
p_{j}=-\frac{\gamma}{2}[(n+1-j)(n-j)+(j-1)(j-2)], \quad q_{j}=-\epsilon \sqrt{j(n+1-j)} .
$$

Hartree energy levels are determined from stationary states of the classical DST, which are discussed in detail in references [1,2]. From eqs. (3.6) and (4.8) one finds that for sufficiently small values of $\gamma$ there are two Hartree levels at

$$
E_{n}^{(\mathbf{H})}=-\frac{1}{4} \gamma n(n-1) \pm \epsilon n,
$$

where the "+" ("-") sign corresponds to an antisymmetric (symmetric) wave function. Above a critical value of the anharmonic parameter, 


$$
\gamma_{c}=2 \epsilon /(n-1),
$$

the symmetric wave function "bifurcates". Thus for $\gamma>\gamma_{\mathrm{c}}$ there are three levels: the two described above plus a localized level for which the expected boson number of the Hartree wave function is larger on one of the two freedoms (see [1,2] for details) and

$$
E_{n}^{(\mathbf{H})}=-\frac{1}{2} \gamma n(n-1)-\left(\frac{n}{n-1}\right) \frac{\epsilon^{2}}{\gamma} .
$$

To appreciate the relationship between the exact wave functions and their Hartree approximations, let us consider the case of two bosons $(n=2)$. From eq. (5.2) the lowest energy eigenvalue (corresponding to a symmetric eigenfunction) has the value

$$
E_{2}^{(s)}=-\frac{1}{2}\left(\gamma+\sqrt{\gamma^{2}+16 \epsilon^{2}}\right) \text {, }
$$

which lies close to the lowest value given by eq. (5.3), for $\gamma\left\langle\gamma_{c}\right.$. For $\left.\gamma\right\rangle \gamma_{c}$ the Hartree level, given by eq. (5.5) lies between the exact value of the lowest (symmetric) level from eq. (5.6) and the next lowest (antisymmetric) level at

$$
E_{2}^{(\mathrm{a})}=-\gamma \text {. }
$$

In this case the Hartree solution is localized [1,2] while exact eigenfunctions of the Hamiltonian operator in eq. (5.1) cannot be because they must share the symmetry of the reflection operator with which $\hat{H}$ commutes. As Bernstein has shown [9], quantum theory responds to this dilemma by producing two lower levels which are quasi-degenerate with splitting

$$
\Delta E_{2} \equiv E_{n}^{(\mathrm{a})}-E_{n}^{(\mathrm{s})}=\frac{2 n \epsilon^{n}}{(n-1) ! \gamma^{n-1}} .
$$

Thus the quantum theory manages to keep energy localized on one freedom for times short compared with the tunneling time: $\hbar / \Delta E_{2}$.

For a more detailed comparison we turn to numerical studies. In fig. 1 the heavy lines indicate the energies of Hartree stationary states that are computed from eqs. (5.3) and (5.5) with $\epsilon=1$ and $n=10$. The light (background) lines are the exact energy eigenvalues obtained from the matrix in eq. (5.2). We note that this plot is similar - but not identical - to one that has recently been prepared by Bernstein to compare classical and exact quantum energies for the DST dimer [10]. Again we see that the lowest Hartree level lies close to the lowest exact eigenvalue; to the accuracy of the plot in fig. 1 they appear identical. The largest of the three Hartree levels lies close to the largest exact level for $\gamma\left\langle\gamma_{\mathrm{c}}\right.$ but diverges for $\gamma>\gamma_{\mathrm{c}}$. An intermediate Hartree level, which appears in fig. 1 for $\gamma>\gamma_{\mathrm{c}}$, is dashed because the corresponding symmetric solution is dynamically unstable $[1,11]$. We see from fig. 1 that this unstable Hartree level indicates where pairs of exact solutions become quasi-degenerate. That is, for $\gamma>\gamma_{c}$ the unstable Hartree branch separates nondegenerate energies (above the dashed line) from quasidegenerate pairs (below). These degenerate pairs allow one to construct wave packets localized to individual freedoms that oscillate about the stationary localized solution for times short compared with the appropriate tunneling times [9].

Next we consider how well the Hartree analysis represents the exact time dependent behavior. Our approach is as follows: $i$ ) We choose an initial condition in eq. (3.6) and compute the time dependent behavior of the Hartree single boson wave function. ii) Eq. (4.2) is then used to obtain the Hartree 


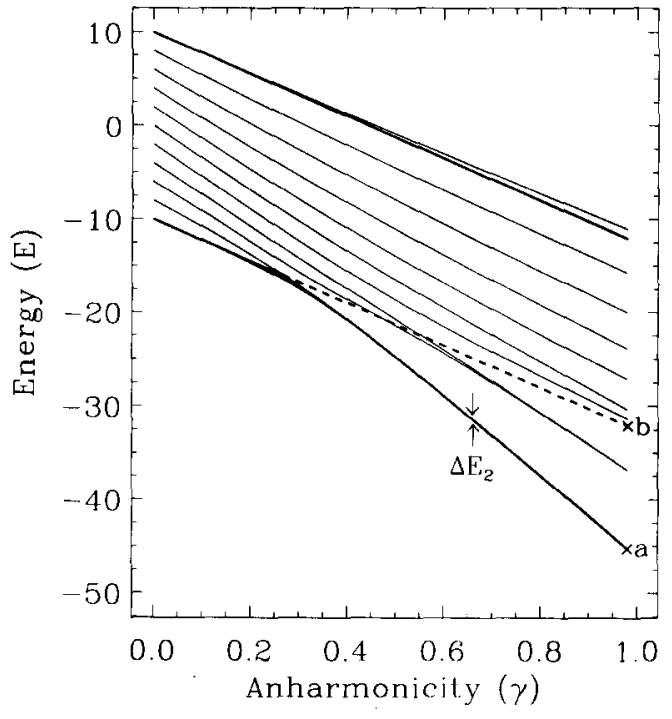

Fig. 1. A comparison of energy level calculations for the quantum DST dimer for two boson $(n=10)$ states with $\epsilon=1$. The heavy solid (dashed) lines are the energies of stable (unstable) Hartree stationary states, which are calculated from eqs. (5.3) and (5.5). The light lines are exact energy eigenvalues computed as eigenvalues of the Hamiltonian matrix in eq. (5.2). To the accuracy of the figure the lowest exact eigenvalue coincides with the lowest Hartree level. The points $x$ indicate initial conditions for time dependent calculations in fig. 2.
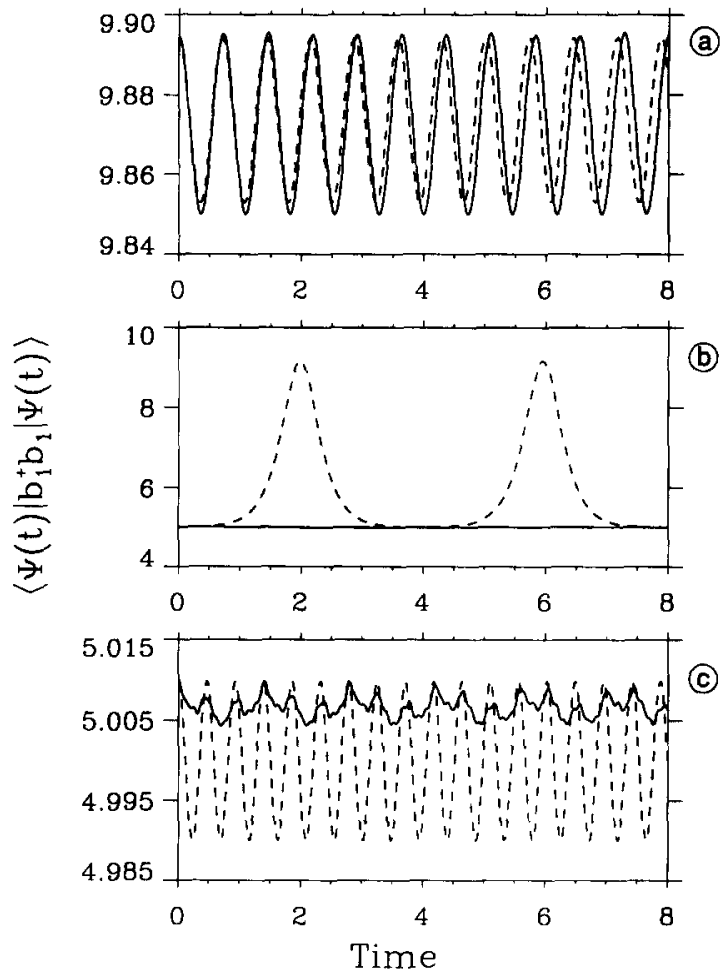

Fig. 2. Comparison of time-dependent Hartree calculations $(----)$ of $\left\langle\psi\left|b_{j}^{\dagger} b_{j}\right| \psi\right\rangle$ with exact calculations (on the DST dimer for three different initial conditions, with $n=10$ and $\epsilon=1$. a) The initial condition is $\gamma=1$ and close to the lowest (local mode) Hartree energy. b) The initial condition is $\gamma=1$ and close to the unstable (symmetric) Hartree branch. c) The initial condition is $\gamma=10$ and close to the highest (antisymmetric) Hartree energy. In this case a larger value of $\gamma$ is chosen to illustrate divergence between Hartree and exact calculations.

estimate of $\left\langle n_{j}(t)\right\rangle^{(\mathrm{H})}$ on a particular freedom. iii) Identical initial conditions for an exact quantum mechanical calculation are obtained from eq. (3.2).

The exact solutions (full lines) and the Hartree estimates (dashed lines) are compared in fig. 2 for initial conditions chosen from three different points in the " $\gamma$-energy" plane of fig. 1 . If the initial conditions are chosen to lie exactly on a Hartree stationary state, then $\left\langle n_{j}(t)\right\rangle$ and $\left\langle n_{j}(t)\right\rangle^{(\mathrm{H})}$ are both constant for all time. Thus we choose initial conditions that are perturbed slightly away from the Hartree stationary states.

The upper part of fig. $2 \mathrm{a}$ is for an initial condition that is close to the localized Hartree solution (see the cross denoted "a" in fig. 1), and the time dependent Hartree behavior is close to that of the exact solution.

Figure $2 \mathrm{~b}$ is initiated at a point that lies close to the unstable Hartree solution in fig. 1, and - in this case - Hartree gives a poor approximation to the exact result. Figure $2 c$ is calculated from an initial condition that is close to the stable antisymmetric branch in fig. 1. If it were plotted for an initial condition with the same value of $\gamma$ as in figs. $2 a$ and $2 b$, the result would be similar to that indicated 
infig. 2a; thus we choose a much larger value of $\gamma$. Here the Hartree solution is periodic while the exact solution is quasiperiodic. This is because the initial condition chosen must be represented by a wave packet with approximately equal contributions from several of the $n+1$ eigenstates.

Finally a note about the time scales in fig. 2. Since we assume $\epsilon=1$, the basic unit of time is $\epsilon^{-1}$. If $\epsilon$ is measured in joules, the time unit will be $h / \epsilon$ seconds. If $\epsilon$ is measured in $\mathrm{cm}^{-1}$ or "wave-numbers", the unit of time is $1 / 2 \pi c \epsilon$, or the time it takes light to travel $(2 \pi \epsilon)^{-1}$ centimeters in a vacuum.

\section{A DST trimer $(f=3)$}

Here we proceed along the lines of the previous section to consider a system with three degrees of freedom: a trimer. Returning to eq. (1.1) we again assume $\omega_{0}-\gamma=0$ and also

$$
m_{j k}=\epsilon\left(1-\delta_{j k}\right),
$$

which implies that each freedom interacts equally with the other two. This example is an interesting generalization of the dimer because it is not classically integrable; thus it has played a role in exploring the relationship between classical and quantum descriptions of chaos [12].

The classical bifurcation diagram for this system has been presented in reference $[1,2]$, and although it can be expressed analytically - it is considerably more complicated than for the dimer case shown in fig. 1. The corresponding Hartree diagram for $\epsilon=1$ and $n=3,5$, and 7 is plotted on the left hand side in fig. 3 from eqs. (3.6) and (4.8), where again the solid (dashed) lines indicate stationary states that are dynamically stable (unstable). Using the number state method exact energy eigenvalues are plotted for the same parameters on the right hand side in fig. 3.

To understand the relationships between exact energy eigenvalues and Hartree stationary energies, the left and right hand sides of figs. 3 should be viewed together. (We suggest that the reader make transparent copies of fig. 3 so the two sets of data can be directly superimposed.) As in the case of the dimer, the lowest Hartree energy is seen to give a good approximation to the lowest (symmetric) energy eigenvalue. Also for $\gamma$ larger than its value at the Hartree bifurcation point, the three lowest levels become quasi-degenerate within an energy range $\Delta E_{3}$ given by Bernstein's formula [9]

$$
\Delta E_{3}=\frac{4 n \epsilon^{n}}{(n-1) ! \gamma^{n-1}} .
$$

As in the case of the dimer, this permits the quantum theory to localize energy on a single freedom for times of order $\hbar / \Delta E_{3}$.

The upper Hartree energy coincides with the highest exact energy eigenvalue at $\gamma=0$ but diverges at increasing values of $\gamma$. Between the highest and lowest Hartree energies are several stationary levels that are unstable as indicated by the dashed lines. These are related in a complicated manner - if at all - to the exact levels. In the vicinity of $\gamma \sim 1$ the statistics of the level spacings is given by the Wigner distribution, which is characteristic of classical chaos [12].

In fig. 4 we compare Hartree and exact calculations of $\left\langle\psi\left|b_{j}^{\dagger} b_{j}\right| \psi\right\rangle$ on the trimer with $\epsilon=1$. The initial conditions are for a Hartree stationary state so the Hartree (dashed) curves are horizontal lines. The exact calculations - on the other hand - oscillate in a quasiperiodic manner as is expected for a quantum mechanical wave packet. In these calculations $\left\langle\psi\left|b_{1}^{\dagger} b_{1}\right| \psi\right\rangle$ shows an oscillation of larger amplitude and $\left\langle\psi\left|b_{2}^{\dagger} b_{2}\right| \psi\right\rangle=\left\langle\psi\left|b_{3}^{\dagger} b_{3}\right| \psi\right\rangle$ is of smaller amplitude. (Note the difference in time scales 

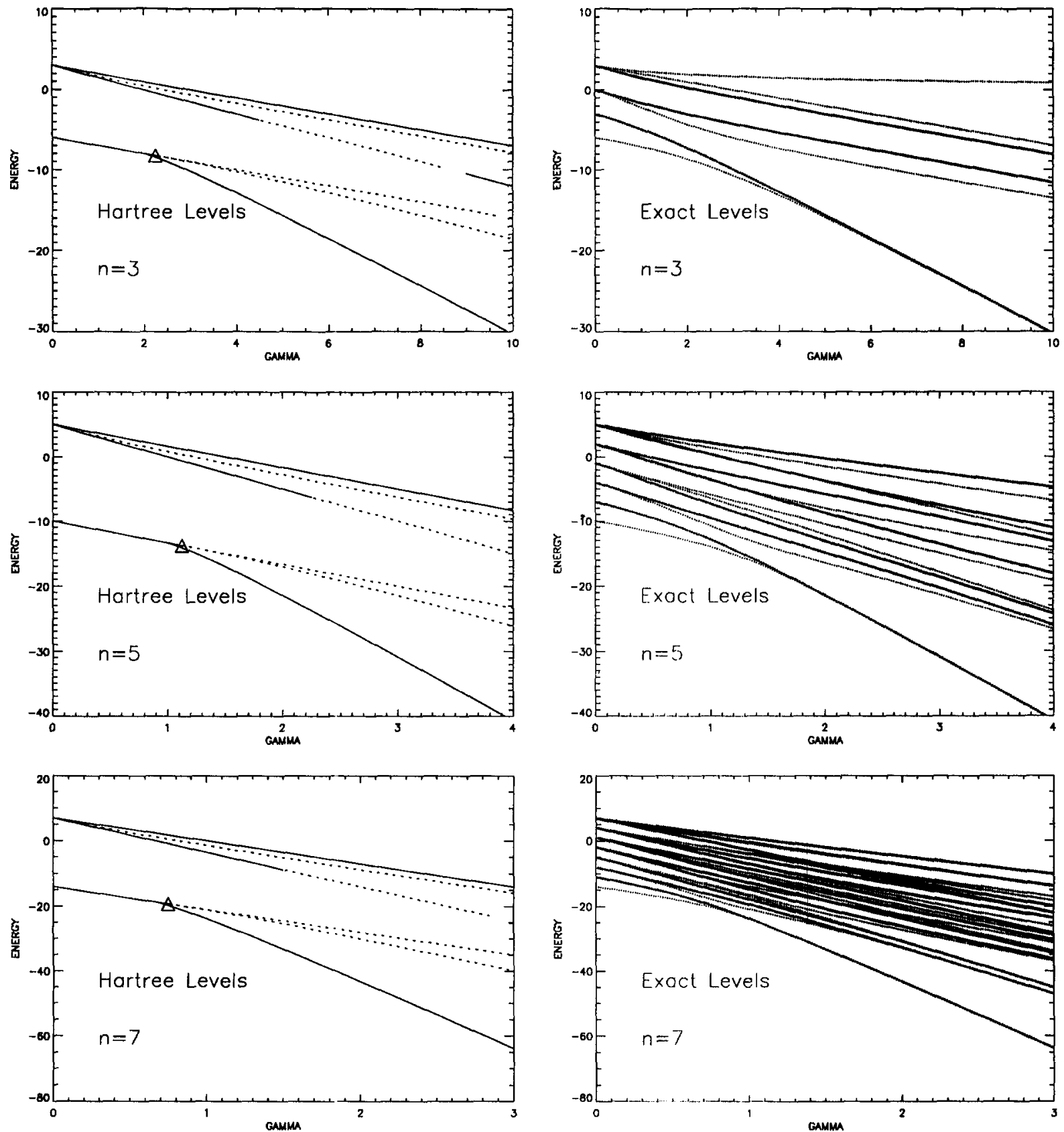

Fig. 3. A comparison of Hartree and exact energy level calculations for the quantum DST trimer for $(n=3,5$, and 7$)$ with $\epsilon=1$. Left hand plots: The energies of Hartree stationary states. The solid (-) lines indicate dynamic stability and the dashed (- - - ) lines indicate dynamic instability. Right hand plots: The exact energy eigenstates calculated for the same parameters. The heavily dotted lines are doubly degenerate (" $E$ " modes) while the lightly dotted lines are non-degenerate ("A" or "B" modes). Left and right hand sides of this figure should be viewed superimposed as in fig. 1. 

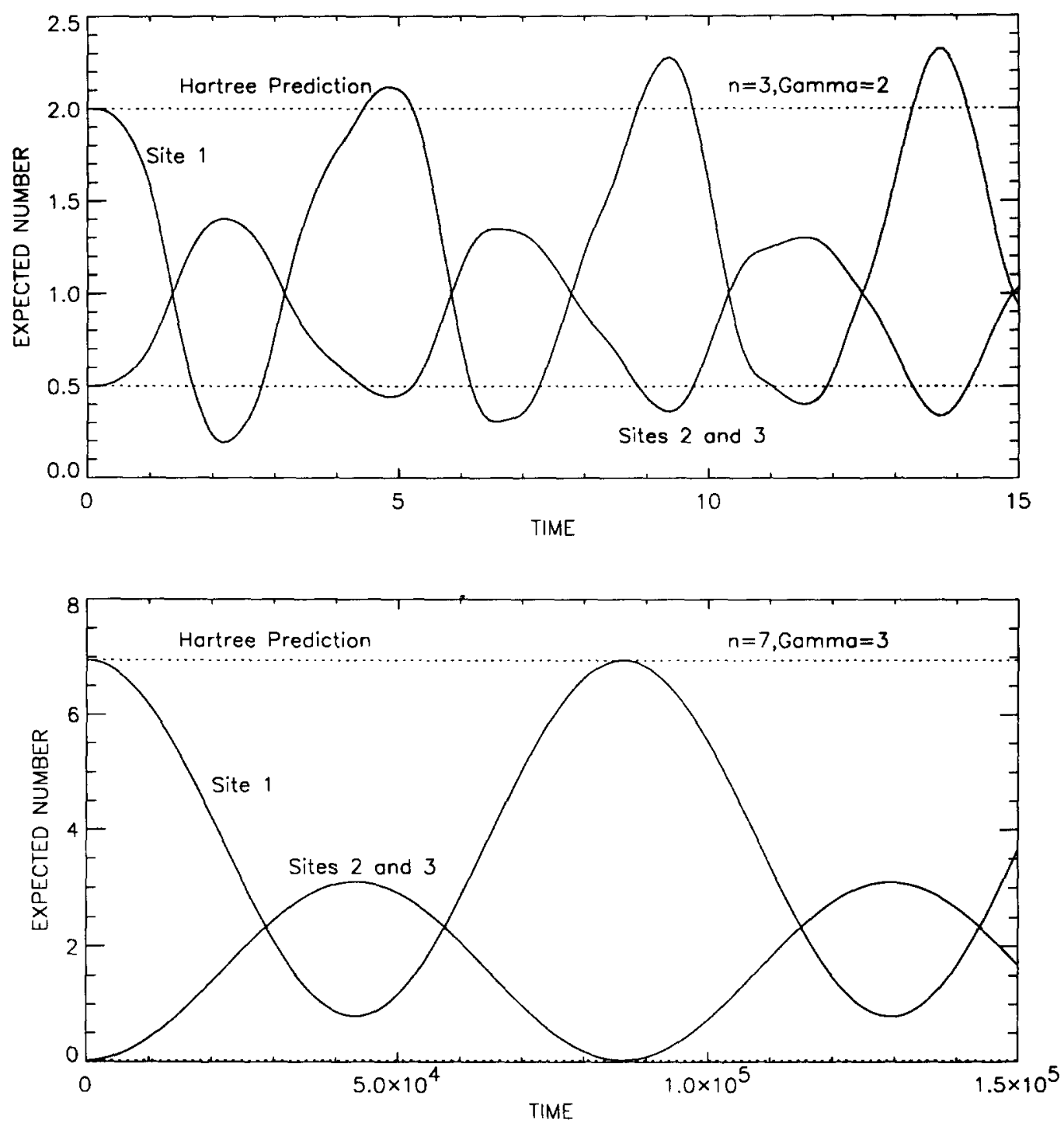

Fig. 4. A comparison of exact (- $\longrightarrow$ ) and Hartree (- - - ) time dependent calculations of $\left\langle\psi\left|b_{j}^{\dagger} b_{j}\right| \psi\right\rangle$ for the DST trimer. The initial conditions for the exact calculations are chosen for Hartree stationary states using eq. (3.2). For the curves of larger amplitude $j=1$, while $j=2,3$ for the curves of lower amplitude.

for the upper and lower plots of fig. 4.) Thus the larger curve plus twice the smaller curve is equal to $n$, the number of bosons. For $n=7$ almost all of the wave packet is composed of the lowest three quantum states, which lie within the range given by eq. (6.2), and the time dependence is almost sinusoidal.

In fig. 5 we present results from a large number of plots of the sort shown in fig. 4. In particular the fundamental frequencies of the exact oscillations are plotted as functions of $\gamma$ for various values of $n$. These calculations are indicated by dots and are compared with $\Delta E_{3}$ from Bernstein's formula in eq. (6.2). Clearly the frequency approaches $\Delta E_{3}$ for the larger values of $\gamma$. Returning to fig. 4 we see that 


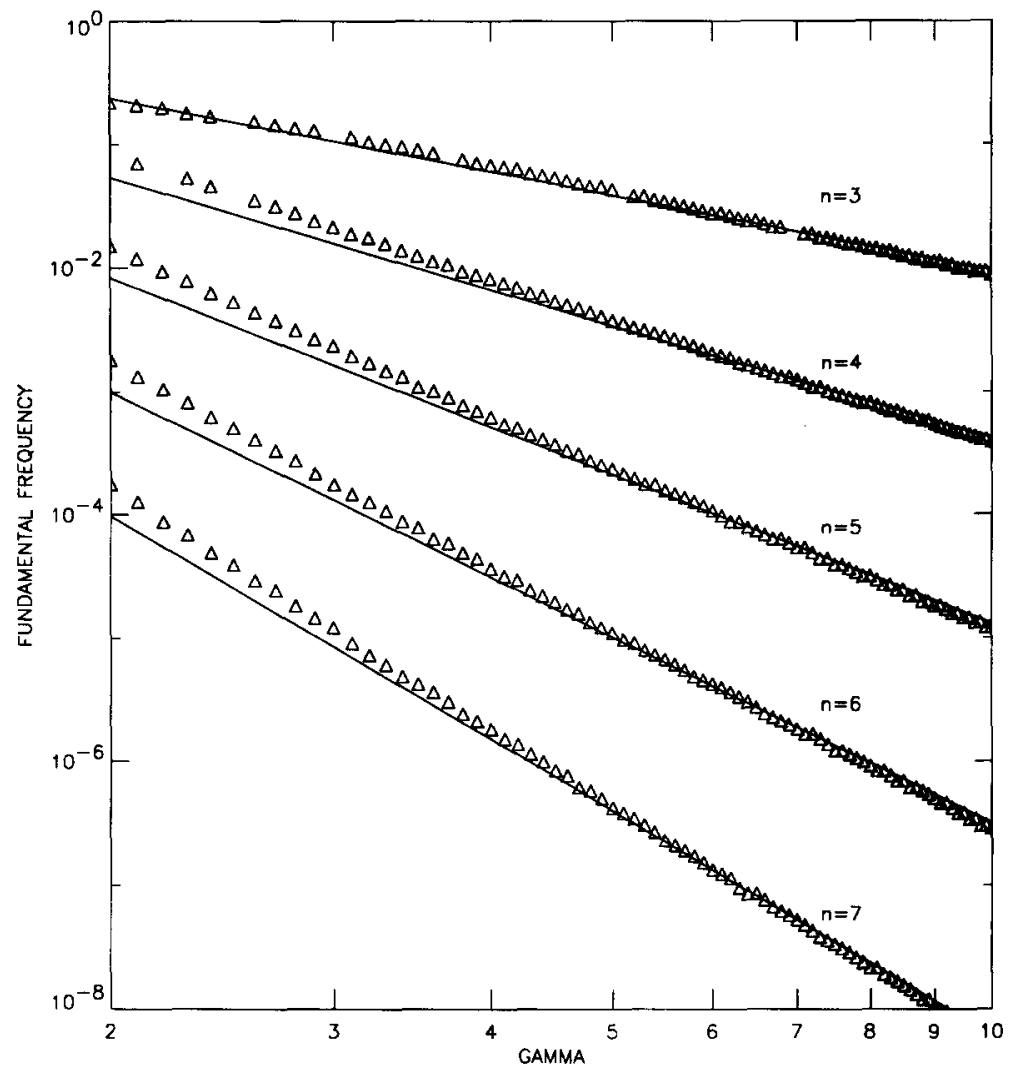

Fig. 5. The dots $(\bullet)$ show fundamental oscillation frequencies from dynamic calculations as are indicated in fig. 4 . The solid lines are computed from Bernstein's formula, which is given in eq. (6.2).

the Hartree approximations remain close to the exact result for times of order $1 / 20$ th of the period or

$$
\tau=\frac{\hbar}{20 \Delta E_{3}} \text { seconds }
$$

if $\Delta E_{3}$ is in joules and $\hbar$ is in joule-seconds. This means that the lowest energy Hartree solution is represented quantum mechanically by a superposition of the three lowest quasi-degenerate levles with energy spacing $\Delta E$, which in turn means that the Hartree solution is only dynamically valid for times $t \leqslant \tau$. Referring to eqs. (6.2) and (6.3), the Hartree approximation improves as $\gamma / \epsilon$ and the number of bosons $n$ increase.

\section{An arbitrary number of freedoms}

The DST dimer and trimer, which we have considered as examples in the previous sections, can be generalized to systems with $f$ freedoms in many different ways: (i) To systems with periodic boundary conditions (so $j+f=j$ ) and

$$
m_{j k}=\epsilon \delta_{j, j \pm 1},
$$

or (ii) To systems with 


$$
m_{j, k}=\epsilon\left(1-\delta_{j, k}\right) .
$$

The interactions indicated in eq. (7.1) are those for a "discrete nonlinear Schrödinger equation" (with nearest neighbor interactions), which has been studied in some detail $[13,14]$. Eq. (7.2) - on the other hand - indicates equal interactions between all $f$ freedoms. Geometrically this might be thought of as a natural model for a regular " $f$-hedron" in a space of $f$-1-dimensions. From a more practical perspective, eq. (7.2) can be considered as a limiting case for a set of oscillators in which the range of interaction is large compared with the size of the system.

For the discrete nonlinear Schrödinger example of eq. (7.1) we make use of results obtained in reference [13], from which it can be shown that the accuracy of the energy of the Hartree ground state depends strongly upon the size of a classical (or Hartree) soliton. In particular if $\gamma$ lies within the range:

$$
\frac{24 \epsilon}{(n+1) f}<\gamma<\frac{24 \epsilon}{(n+1)},
$$

the continuum approximation holds and the exact soliton binding energy is [15]

$$
E_{\mathrm{B}}=\frac{\gamma^{2}}{48 \epsilon} n\left(n^{2}-1\right)
$$

while the corresponding Hartree approximation is [16]

$$
E_{\mathrm{B}}^{(\mathrm{H})}=\frac{\gamma^{2}}{48 \epsilon} n(n-1)^{2} .
$$

Thus within the range of eq. (7.3) the Hartree method underestimates soliton binding energies by the factor $(n-1) /(n+1)$. Outside the range indicated in eq. (7.3) the Hartree approximation gives a good estimate of the ground state energy.

Turning to the strongly interacting (or complete graph) system of eq. (7.2) we consider the case of two bosons $(n=2)$ for which the NSM gives the lowest exact energy eigenvalue as

$$
E_{2}=-(f-2)-\frac{1}{2} \gamma-\sqrt{\left(f \epsilon-\frac{1}{2} \gamma\right)^{2}+2 \gamma} .
$$

For the classical DST we recall that the $(\uparrow)^{f}$ stationary solution was obtained in [1] as $\Phi_{j}=1 / \sqrt{f}$; thus the single boson energy is

$$
e_{2}=-\frac{\gamma}{2 f}-\epsilon(f-1)
$$

There is a bifurcation point at $(\gamma, e)=\left(\frac{1}{2} \epsilon f^{2}, \epsilon\left(-\frac{5}{4} f+1\right)\right)$. The $\uparrow(\cdot)^{f-1}$ (soliton) stationary solution was given parametrically by [1]

$$
\omega+1=\frac{3\left(f^{2}-3 f+3\right)^{1 / 2} \sin \left(\theta+\theta_{0}\right)}{\sin 3 \theta},
$$




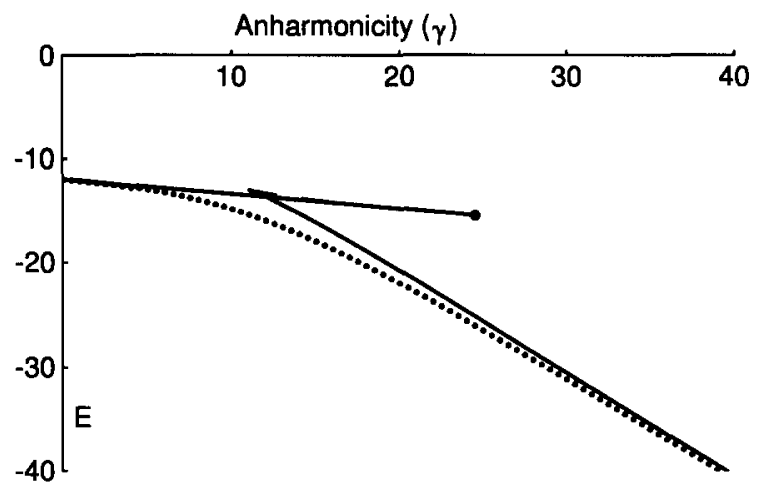

Fig. 6. A comparison of the exact $(\cdots \cdots \cdots)$ lowest energy eigenfunction for the strongly interacting (or complete graph) system with the corresponding stable Hartree approximation (-) for $f=7, n=2$ and $\epsilon=1$. An unstable Hartree branch is indicated by the dashed (- - - -) line. Exact energies are calculated from eq. (7.6) and the Hartree approximations from eq. (7.9) using eq. (7.7) along the left hand branch and eq. (7.8) along the right hand branch.

$$
e_{2}=-\frac{1}{2 \gamma}\left[\psi_{1}^{4}+(f-1) \psi_{2}^{4}\right]-\frac{f-1}{\gamma}\left[2 \psi_{1} \psi_{2}+(f-2) \psi_{2}^{2}\right]
$$

From eq. (4.8) the total Hartree energy is

$$
E_{2}^{(H)}=2 e_{2}
$$

In fig. 6 we assume $f=7$ and $\epsilon=1$ and compare the exact value of the lowest energy eigenvalue from eq. (7.6) with the corresponding Hartree approximations calculated from eq. (7.10) along the two branches defined by eqs. (7.7) and (7.8). The dotted line shows the exact energy as a function of $\gamma$, and the solid lines indicate the Hartree approximation. Note that the lowest energy Hartree solution jumps from a $(\uparrow)^{f}$ to a $\uparrow(\cdot)^{f-1}$ solution near $(\gamma, E)=(11,13)$.

\section{Summary and conclusions}

In this paper we have shown how to construct the Hartree approximation (HA) to the $n$-boson wavefunction for the quantum discrete self-trapping (QDST) equation with $f$ freedoms and arbitrary linear interactions. This is a useful approximation because it reduces the order of the quantum problem to that of the corresponding nonlinear classical problem.

From investigations of the QDST dimer, trimer, and $f$-mer, we draw the following conclusions:

- For a small number of freedoms the energy of the lowest Hartree stationary state gives a good approximation to the lowest exact energy eigenvalue.

- For a small number of freedoms the difference between the lowest and highest energy levels in the Hartree approximation gives a reasonable estimate of the bandwidth of the exact energy eigenvalues.

- Time dependent Hartree calculations from initial conditions on the lowest stationary state - for $\gamma>\epsilon$ and a small number of freedoms - are in good agreement with exact calculations for times of order $\hbar / \Delta E_{f}$, where $\Delta E_{f}$ is the splitting of the $f$ lowest (quasi-degenerate) energy eigenvalues.

- For the discrete nonlinear Schrödinger equation with periodic boundary conditions and $f \gg 1$, studies of the lowest Hartree and exact energies indicate a maximum error in binding energy of about $200 / n \%$ for nearest neighbor interactions, where $n$ is the number of bosons. 


\section{Acknowledgements}

We thank Lisa Bernstein for a careful reading of the manuscript and acknowledge support from the Joint Services Optical Program, from the SERC Nonlinear Systems Initiative and the EC under SCI0229-C89-100079/JU1, and from the National Science Foundation under Grant No. DMS-9114503.

\section{References}

[1] J.C. Eilbeck, P.S. Lomdahl and A.C. Scott, Physica D 16 (1985) 318.

[2] J.C. Eilbeck in Davydov's soliton revisited, P.L. Christiansen and A.C. Scott, eds. (Plenum, N.Y.,1990) p. 473.

[3] A.C. Scott and J.C. Eilbeck, Chem Phys. Lett. 132 (1986) 23.

[4] A.C. Scott and J.C. Eilbeck, Phys. Lett. A 119 (1986) 60.

[5] V.Z. Enol'skii, M. Salerno, A.C. Scott and J.C. Eilbeck, Physica D 59 (1992) 1.

[6] B. Yoon and J.W. Negele, Phys. Rev. A 16 (1977) 1451; J.W. Negele, Rev. Mod. Phys. 54 (1982) 913.

[7] For a discussion of the corresponding quantum field theory see, e.g., K. Huang, Statistical Mechanics, second ed. (Wiley, New York, 1987), Appendix A.

[8] Y. Lai and H.A. Haus, Phys. Rev. A 40 (1989) 844;

E.M. Wright, Phys. Rev. A 43 (1991) 3836.

[9] L. Bernstein, J.C. Eilbeck and A.C. Scott, Nonlinearity 3 (1990) 293.

[10] L. Bernstein, Quantizing a self-trapping transition, Physica D (to appear).

[11] J. Carr and J.C. Eilbeck, Phys. Lett. A 109 (1985) 201.

[12] S. de Filippo, M. Fusco Girard and M. Salerno, Physica D 26 (1987) 411; 29 (1988) 421; Nonlinearity 2 (1989) 477; L. Cruzeiro-Hansson, H. Feddersen, R. Flesch, P.L. Christiansen, M. Salerno and A.C. Scott, Phys Rev. B 42 (1990) 522.

[13] P.D. Miller, A.C. Scott, J. Carr and J.C. Eilbeck, Physica Scripta 44 (1991) 509.

[14] J.C. Eilbeck, H. Gilhøj and A.C. Scott, Phys. Lett. A 172 (1992) 229.

[15] A. Klein and F. Krejs, Phys Rev. D 13 (1976) 3282;

M. Wadati, A. Kuniba and T. Konishi, J. Phys. Soc. Japan 54 (1985) 1710.

[16] F. Calogero and A. Degasperis, Phys. Rev. A 11 (1975) 265. 